\title{
Cloning and Expression of Omission of Second Division-like Genes from Carnation
}

\author{
Xuhong Zhou, Xijun Mo, Yalian Jiang, Hao Zhang, Rongpei Yu, Lihua Wang, Jihua Wang ${ }^{1}$ \\ and Suping $\mathbf{Q u}{ }^{1}$ \\ Flower Research Institute, Yunnan Academy of Agricultural Science, Kunming 650205, China; \\ and National Engineering Research Center for Ornamental Horticulture, Kunming 650205, China
}

\begin{abstract}
AdDITIONAL INDEX words. Dianthus caryophyllus, OSD1, meiosis, 2n pollen, mechanism, polyploidization
Abstract. The omission of second division gene (OSD1) gene plays a fundamental role in meiosis and is associated with 2n gamete formation in Arabidopsis thaliana. The objective of this work was to unravel the mechanisms leading to $2 \mathrm{n}$ pollen production, and isolate and analyze the expression patterns of $O S D$-like (OSDL) genes in carnation (Dianthus caryophyllus). We found an absence of the second meiotic division caused the formation of $2 \mathrm{n}$ pollen. Three homoeologous genes were cloned and labeled as $O S D L a, O S D L b$, and $O S D L c$ in a diploid carnation. The cDNAs were $1180 \mathrm{bp}$ for $O S D L a, 1288$ bp for $O S D L b$, and $971 \mathrm{bp}$ for $O S D L c$. A strong similarity was found between the amino sequences of $O S D L b$ and $O S D L c$. An evident feature of $O S D L$ s proteins is the presence of D-box and MR-tail domains; however, the GxEN/KEN-box domain, which is distinct among the other plant proteins was absent. Quantitative real time polymerase chain reaction (qRT-PCR) analysis showed that $O S D L$ genes maintain continuous expression in buds and other tissues. $O S D L a$ has the highest expression in buds of 1.1-1.2 cm long (stage 2), and $O S D L b$ has a high level of expression in buds of $0.9-1.0 \mathrm{~cm}$ long (stage 1) and stage 2 buds and ovary tissues in three carnation cultivars. The expression level of $O S D L c$ was highest in ovaries. These expression patterns strongly suggest that $O S D L s$ in carnation involve male meiosis and ovary development. These findings can have potential applications in fundamental polyploidization research and plant breeding programs in carnation.
\end{abstract}

Meiosis is a specialized cell division essential for sexual reproduction. It involves a single DNA replication event followed by two rounds of chromosome segregation- meiosis I (M I) and meiosis II (M II) - to produce cells with half the chromosome number of the mother cell. Thus, to generate haploid spores, a meiocyte must enter M I after prophase, pass through the M I to M II transition, and complete M II. Errors in transitions are not uncommon and may lead to the production of viable, $2 \mathrm{n}$ gametes, as have been described in a range of plants (Bretagnolle and Thompson, 1995; Ramanna and Jacobsen, 2003). Unreduced gamete is now believed to be the major route for polyploidization through sexual reproduction. Polyploidy has played a key role in the evolution of many fungal, plant, invertebrate, and vertebrate lineages, and is particularly frequent in plants (Otto, 2007; Otto and Whitton, 2000). 2n gametes are also an important tool for plant breeding (Ramanna and Jacobsen, 2003). However, despite the evolutionary and agricultural significance of sexual polyploidization in plants, the underlying genes have not been fully characterized.

Unreduced gamete formation derived via abnormal meiotic cell division is an important phenomenon for polyploid breeding (Ramanna and Jacobsen, 2003). More than 10 abnormal mechanisms leading to $2 \mathrm{n}$ gamete formation have been reported to date (Bretagnolle and Thompson, 1995). Within the same species, several abnormal cytogenetic events can lead to the formation of the 2n gametes (Gómez-Rodríguez et al., 2012;

Received for publication 14 Mar. 2017. Accepted for publication 24 Aug. 2017. This work was supported by grants from the National Engineering Research Center for Ornamental Horticulture (grant number 2012FU125X10), Chinese Natural Science Foundation (grant number 31460530), Yunnan Foundation Research Projects for Application (grant number 2014FA044), Yunnan Young Academic and Technical Leader Training (grant number 2015HB077), and Yunnan Science and Technology Leader Training (grant number 2016HA005). ${ }^{1}$ Corresponding authors. E-mail: wjh0505@gmail.com or qsp2002@sohu.com.
Teoh, 1984). Teoh reported four mechanisms that resulted in $2 \mathrm{n}$ gamete formation in orchid species belonging to the Arethuseae tribe (Teoh, 1984): 1) premeiotic disturbances, 2) faulty anaphase disjunction, 3) failure to participate in M I or M II, and 4) co-orientation of the two spindles during metaphase II. In Agave tequilana and Agave angustifolia, two different mechanisms led to $2 \mathrm{n}$ gamete formation: failure of cytokinesis in M II and abnormal spindles (Gómez-Rodríguez et al., 2012). Four mechanisms of $2 \mathrm{n}$ pollen formation observed in diploid potato (Solanum tuberosum) are termed parallel spindles, premature cytokinesis 1, premature cytokinesis 2 (Mok and Peloquin, 1975), and desynapsis (Ramanna, 1983; Veilleux, 1985).

Entry into either mitosis or meiosis requires high cyclindependent kinase $(C D K)$ activity, which is achieved by cyclins of the A and B groups in A. thaliana (Francis, 2007; Inzé and De Veylder, 2006; Menges et al., 2005). Anaphase progression is regulated by a gradual decline in $C D K$ activity, and mitotic program exit requires low $C D K$ activity (de Gramont and Cohen-Fix, 2005). $C D K$ activity is regulated by the anaphase promoting complex/cyclosome $(A P C / C)$, a conserved multisubunit E3 ubiquitin ligase, during mitosis and meiosis. Depletion of $C D K$ activity during meiosis needs to be tightly coordinated, so that the remaining $C D K$ level at the end of M I enables exit from M I, but still allows initiation of M II (Furuno et al., 1994; Futcher, 2008; Iwabuchi et al., 2000; Marston and Amon, 2004; Stern, 2003). Relatively small modifications of the cell cycle during M I or M II may offer the potential to create diploid gametes (Brownfield and Köhler, 2011).

A gene involved in the M I to M II transition is the $A$. thaliana OSDI/GIG1 (gigas cell 1) gene. It has been proposed that $O S D 1$ may inhibit the activity of the $A P C$, which would promote $C D K$ activity (d'Erfurth et al., 2009). Thus, because of the loss of $O S D 1$, a moderate decrease in $C D K$ activity may 
prevent entry into M II without impairing the prophase to M I transition. As osd 1 mutants fail to enter the second division in both male and female meiosis, functional $2 \mathrm{n}$ gametes and tetraploid progeny are produced (d'Erfurth et al., 2009). Male meiocytes lacking both $C Y C A 1 ; 2 / T A M$ and $O S D 1$ fail to enter the first meiotic division (d'Erfurth et al., 2010). Loss of both $C Y C A 1 ; 2 / T A M$, and $O S D 1$ may cause a loss of $C D K$ activity sufficient to impair entry into $\mathrm{M}$ I. These differences highlight the essential and tight regulation of $C D K$ activity required during meiosis (Brownfield and Köhler, 2011).

The $A$. thaliana OSDI has the same three putative cell-cyclerelated domains. These three domains are very well conserved over OSD1 homologues (d'Erfurth et al., 2009). Two of these domains are putative $A P C / C$ degradation motifs: a D-box (residues 104-110, RxxLxx) and a GxEN/KEN-box (residues 80-83, GxEN in eudicotyledon and KEN in monocotyledon OSD1 homologues) (Cromer et al., 2012). OSD1 also has a Cterminal MR-tail in common (the two last amino acid of the protein are a methionine and an arginine) (Cromer et al., 2012). Both the interactions with $A P C / C$ and in-plant meiotic function of OSD1 are dependent on its D-box domain and its MR tail. Remarkably, the OSD1 GxEN-box is not required for OSD1 function, but its mutation allows the $O S D 1$ protein mutated in its D-box to fulfill its function (Cromer et al., 2012).

Interestingly, OSD1 has a paralogue, $u v$-insensitive4 (UVI4), which negatively regulates endoreplication onset in $A$. thaliana that play roles in the mitotic cell cycle through $A P C / C$ regulation (Heyman et al., 2011; Pesin and Orr-Weaver, 2008). However, OSD1 also has a somatic function as revealed by the mitotic phenotype of the single osdl mutants which trigger ectopic endomitosis (Iwata et al., 2011). Iwata et al. (2011) showed that GIG1/OSD1 and UVI4 encode novel plant-specific inhibitors of $A P C / C$ ubiquitin ligase.

Carnation is a major floricultural crop in China and worldwide. It is a member of the Caryophyllaceae. Most carnation cultivars can produce $2 \mathrm{n}$ gametes, and the frequency of $2 \mathrm{n}$ pollen produced ranged from $0 \%$ to $4.17 \%$ (Zhou et al., 2012, 2015). Spindle abnormalities during metaphase II have been considered to be the major cytological mechanism involved in the formation of $2 \mathrm{n}$ pollen in carnation (Zhou et al., 2015). In this study, we describe another abnormality mechanism and found some cells were normal in the first meiotic division; however, a failure occurred in the second meiotic division, which generated dyads during microsporogenesis. The carnation $O S D 1$ gene may be involved in the formation of $2 \mathrm{n}$ gametes. The role of carnation $O S D L$ in the formation of unreduced gametes is described herein through the isolation, characterization, and analysis of expression patterns.

\section{Materials and Methods}

Plant materials. The experiments were carried out in four diploid carnation $(2 \mathrm{n}=30)$ cultivars: Promesa, Guernse Yellow, Nogalte, and YunhongErhao, which displayed
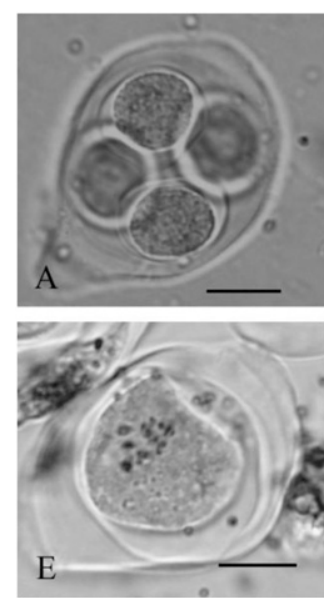
dyad; bars $=10 \mu \mathrm{m}$. high pollen viability. The frequency of dyad production in 'Nogalte' in June 2013 was 3.3\%, and the frequency of $2 \mathrm{n}$ pollen was $1.63 \%$ (Zhou et al., 2015). The percentage of $2 \mathrm{n}$ pollen in 'Promesa', 'Guernse Yellow', and 'YunhongErhao' was $0.06 \%, 3.09 \%$, and $1.72 \%$, respectively (Zhou et al., 2015). They were cultivated under plastic in an ambient light and temperature regimen in Sept. 2012 at Jinning district, in Kunming, Yunnan, China. During the flowering period (June 2013), the daily temperature averaged $22.84 \pm 0.02{ }^{\circ} \mathrm{C}$, and the photoperiod averaged $13 \mathrm{~h}$.

Cytology. 'Nogalte' was used for cytological analysis of male meiosis. Observation of the final male meiotic products and chromosome spreads were carried out as previously described (Zhou et al., 2015), observed under a light microscope (E800; Nikon, Tokyo, Japan) at $\times 1000$.

Three carnation cultivars Promesa, Guernse Yellow, and YunhongErhao were sampled in June 2013. Buds were classified into eight stages (1-8) in accordance with the methods described by Zhou et al. (2015). Stage 1 and stage 2 buds usually have developed microspore mother cells (bud size 0.91.0 and $1.1-1.2 \mathrm{~cm}$, respectively); stage 3 buds contain anthers that have generally entered meiosis (bud size 1.3-1.4 cm); stage 4 buds have developed tetrads (bud size 1.5-1.6 cm); and stages 5-8 have further progressed to the mature pollen stages (bud size $1.7-2.4 \mathrm{~cm})$.

Carnation OSDL Cloning. An $A$. thaliana OSD1 cDNA sequence was retrieved from the GenBank database (Benson et al., 1997). A homology search was conducted with BLASTX on the DNA Data Bank of Japan (DDBJ) website (Tanase et al., 2012). Accession numbers in D. caryophyllus FX309965, FX311539, FX311540, FX315550, and FX332100 resulted from this search (Tanase et al., 2012). Fragments of accession numbers FX311540 and FX311539 could be assembled into a 1262-bp segment with a polyA; FX315550 and FX332100 could be assembled into 758-bp segment with a polyA; and FX309965 was 681-bp long. These three putative homologous genes in D. caryophyllus were named OSDLa, OSDLb, and OSDLc, respectively. Total RNA from prebolting buds of 'Promesa' was extracted with TRIZOL Reagent (Invitrogen, Carlsbad, CA) following the manufacturer's instructions. Total
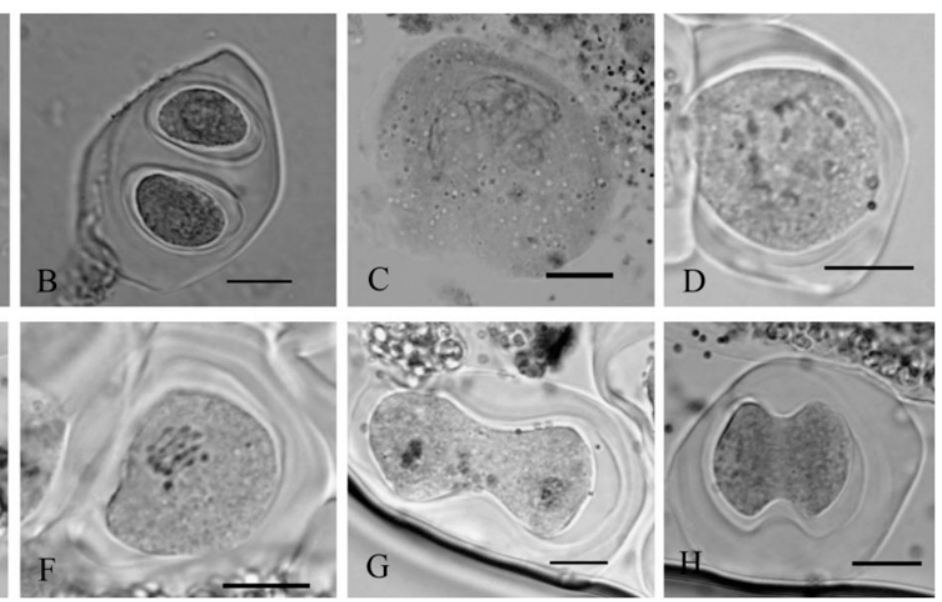

Fig. 1. Abnormal meiosis lacking meiosis II in carnation: (A) a wild-type tetrad; (B) a dyad; (C-H) male abnormal meiosis is indistinguishable from wild type until telophase I, but no figures characteristic of a second division were observed; (C) pachytene; (D) diakinesis; (E) metaphase I; (F) anaphase I; (G) telophase I; (H) an incipient 
RNA was reverse transcribed to the first-strand cDNA with an oligo (dT) primer designed with an adaptor sequence according to the protocol of the SMART RACE cDNA Amplification Kit (Clontech, Mountain View, CA). To isolate the 5' -ends of the carnation $O S D L a, O S D L b$, and $O S D L c$ cDNA, gene-specific primers, OSDL1a-5Ra-364R, OSDL1b-5Ra-189R, and OSDL1c-5R-468R (Supplemental Table 1) were used in combination with a universal primer mix (UPM) for 5'-rapid amplification of cDNA ends (RACE), respectively. The carnation $O S D L a$ and $O S D L b$ cDNA were first amplified. Initial $5^{\prime}$ RACE products of $O S D L c$ gene were then used for nested polymerase chain reaction (PCR) amplifications by using a gene-specific, nested primer [OSDL1c-328R (Supplemental Table 1)], in combination with a nested universal primer (NUP). To isolate the $3^{\prime}$-ends of the carnation OSDLa, OSDLb, and $O S D L c$ cDNA, gene-specific primers, OSDL1a-82-length-F, OSDL1b-138-length-F, and OSDL1c-69-length-F (Supplemental Table 1) were used, respectively. The initial 3 '-RACE products of $O S D L a, O S D L b$, and $O S D L c$ were then used for nested PCR amplifications by using gene-specific nested primers, OSDL1a-103-length-F, OSDL1b-147-length-F, and OSDL1c-113-length-F (Supplemental Table 1). Both the UPM and NUP were supplied with the SMART RACE cDNA amplification kit (Clontech). All RACE reactions were carried
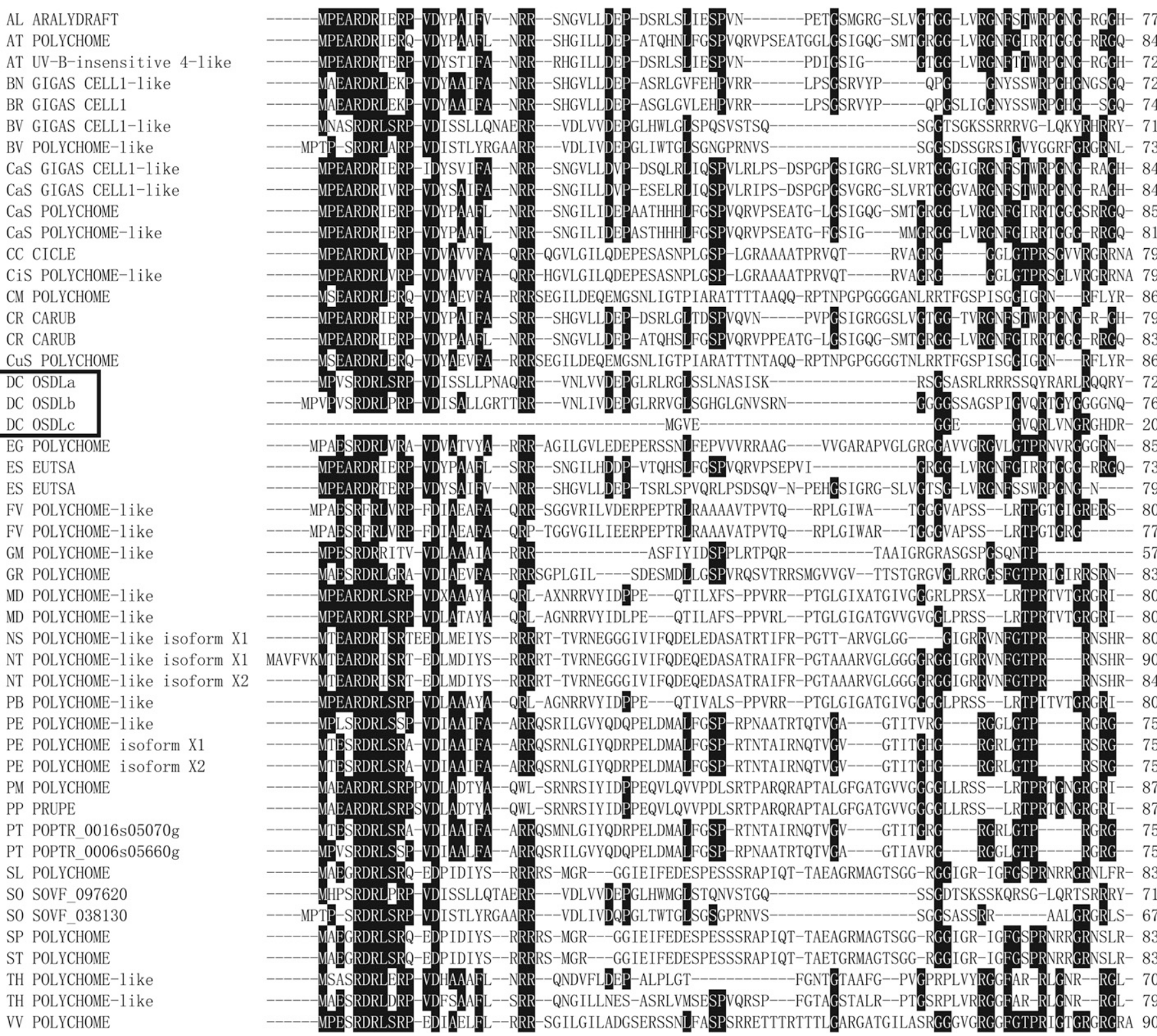

GLHWLGLSPQSVSTSQ--
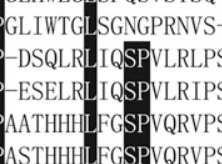

ASTHHHLFG

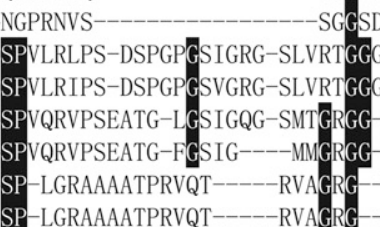
\begin{tabular}{l} 
GGTSGKSSRRRVG-LQKYRHARY- 71 \\
\hline
\end{tabular}

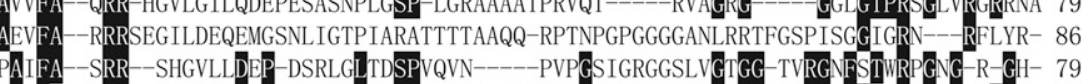

-GGLGTPRSGLVRGRRA 79
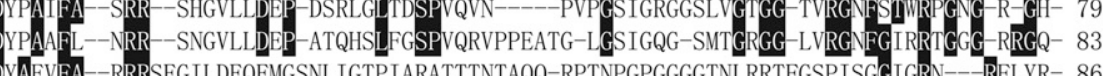

AEVFA--RRRSEGILDEQEMGSNLIGTPIARATTTNTAQQ-RPTNPGPGGGGTNLRRTFGSPISGGIGRN---RFLYR- 86 SSLLPNAQRR --VNLVVDEPGLRLRGLSSLNASISK---_-_-_RSGSRLRRRSQYRARLRQQRY- 72

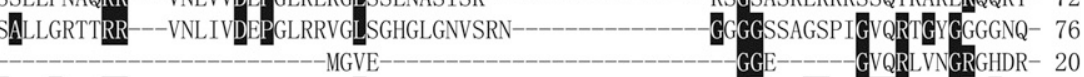

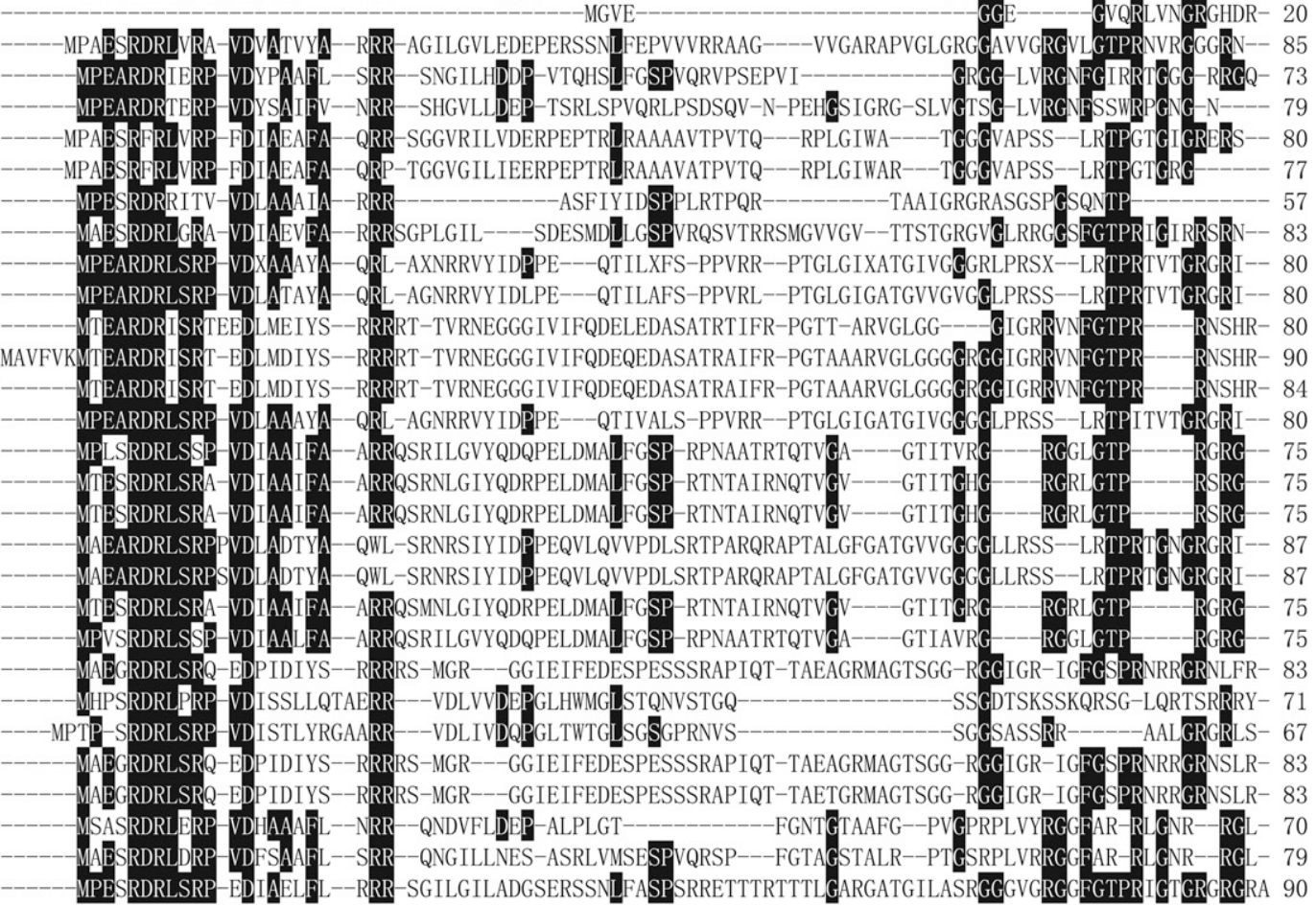

Fig. 2. Alignment of protein carnation omission of second division-like (OSDL) sequences obtained from the National Center for Biotechnology Information online Web tool. The conserved D-box and MR tail motifs are indicated. $\mathrm{AL}=$ Arabidopsis lyrata $\mathrm{ssp}$. lyrata, $\mathrm{AT}=$ A. thaliana, $\mathrm{BN}=$ Brassica napus, $\mathrm{BR}=$ Brassica rapa, $\mathrm{BV}=$ Beta vulgaris ssp. vulgaris, $\mathrm{CC}=$ Citrus clementina, $\mathrm{CM}=$ Cucumis melo, $\mathrm{CR}=$ Capsella rubella, $\mathrm{CaS}=$ Camelina sativa, $\mathrm{CiS}=$ Citrus sinensis, $\mathrm{DC}=$ Dianthus caryophyllus, $\mathrm{EG}=$ Eucalyptus grandis, $\mathrm{ES}=$ Eutrema salsugineum, $\mathrm{FV}=$ Fragaria vesca, $\mathrm{GM}=$ Glycine max, $\mathrm{GR}=$ Gossypium raimondii, $\mathrm{MD}=$ Malus domestica, $\mathrm{NS}=$ Nicotiana sylvestris, $\mathrm{NT}=$ Nicotiana tomentosiformis, $\mathrm{PB}=$ Pyrus bretschneideri, $\mathrm{PE}=$ Populus euphratica, $\mathrm{PM}=$ Prunus mume, $\mathrm{PP}=$ Prunus persica, $\mathrm{PT}=$ Populus trichocarpa, $\mathrm{SL}=$ Solanum lycopersicum, $\mathrm{SO}=$ Spinacia oleracea, $\mathrm{SP}=$ Solanum pennellii, $\mathrm{ST}=$ Solanum tuberosum, $\mathrm{TH}=$ Tarenaya hassleriana, $\mathrm{VV}=$ Vitis vinifera . 
out according to the manufacturer's instructions with Advantage 2 polymerase (Clontech). RACE products were subcloned into a pMD18-T vector (TaKaRa, Kyoto, Japan) and checked for the correct orientation by DNA sequence analysis. Sequencing and phylogenetic analysis were carried out as previously described (Zhou et al., 2015). Theoretical molecular weights of each ribosomal protein were calculated using the Compute pI/Mw tool (Gasteiger et al., 2003).

Phylogenetic analysis. After obtaining the carnation OSDLs amino acid sequences, a BLAST search for homology was performed at the National Center for Biotechnology Information [NCBI (Benson et al., 1997)]. A phylogenetic tree was constructed with MEGA 5.1 (Tamura et al., 2011) based on the converted result from BIOEDIT alignments. The construction of a phylogenetic tree was performed using maximum likelihood. The robustness of clustering was evaluated by bootstrap on 1000 replications.

qRT-PCR ANALYSIS. $O S D L$ expression profiles in various organs (roots, stems, leaves, and ovaries) and developmental stages of buds were investigated using qRT-PCR in carnation 'Promesa', 'Guernse Yellow', and 'YunhongErhao'. Total RNA was isolated and reverse transcribed to the first-strand cDNAs following the manual of the PrimeScript RT reagent Kit with gDNA Eraser (TaKaRa). The GAPDH gene (GAPDH 579$\mathrm{F}$ and GAPDH 788-R) was used as an internal control in all qRT-PCR reactions (Supplemental Table 1). Real time polymerase chain reaction (RT-PCR) was carried out on $50 \mathrm{ng}$ of cDNA with the OSDL1a-422F and OSDL1a-656R primers for OSDLa, the OSDL1b-99-F, and OSDL1b-223-R primers for $O S D L b$, and the OSDL1c-133F and OSDL1c-270-R primers

\section{GxEN-box D-box}

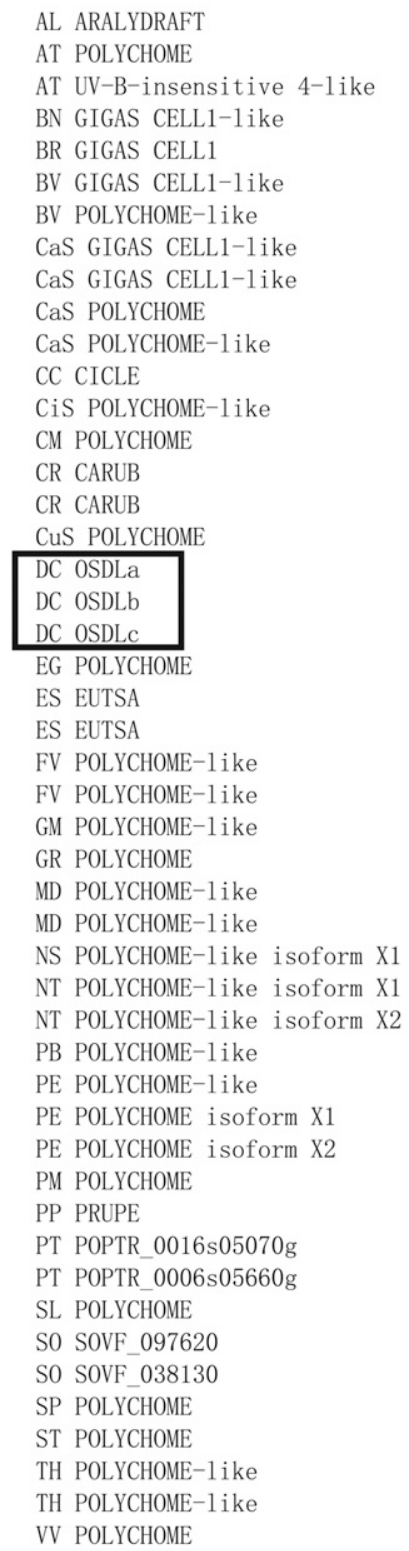

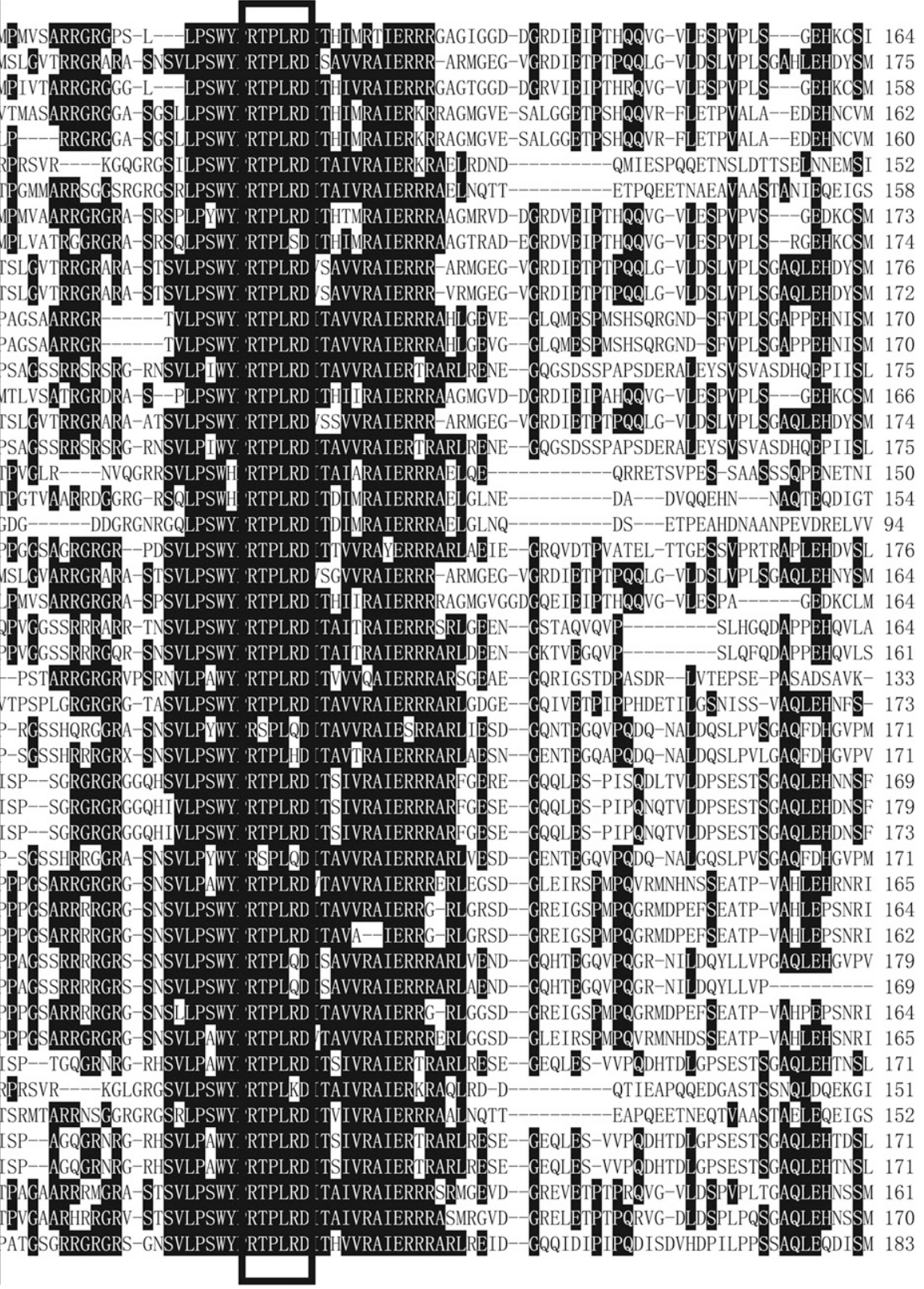


for OSDLc (Supplemental Table 1). Gene expression in different tissues was characterized by using the Faststart SYBR Green Master Mix kit (Roche, Mannheim, Germany) following the manufacturer's instructions. Each result reported herein resulted from three biological replicates. For each sample, quantification was made in triplicate (technical replicates). Three plants for each cultivars with different sizes of buds were selected which were grown and arranged in the same growing area. Values are means \pm SE of three replicates. Different letters indicate significant differences $(P<0.05)$. Data were subjected to analysis of variance with the least significant difference method by statistical software package SPSS 18 (IBM Corp., Armonk, NY).

\section{Results}

The Cytological Mechanism Leading to 2N POLlen PRODUCTION AND 2N FREQUENCY. To unravel the mechanisms leading to $2 \mathrm{n}$ pollen production, we investigated chromosome behavior during meiosis. During microsporogenesis in 'Nogalte', subsequent reductional and equational divisions normally result in the formation of a group of four haploid

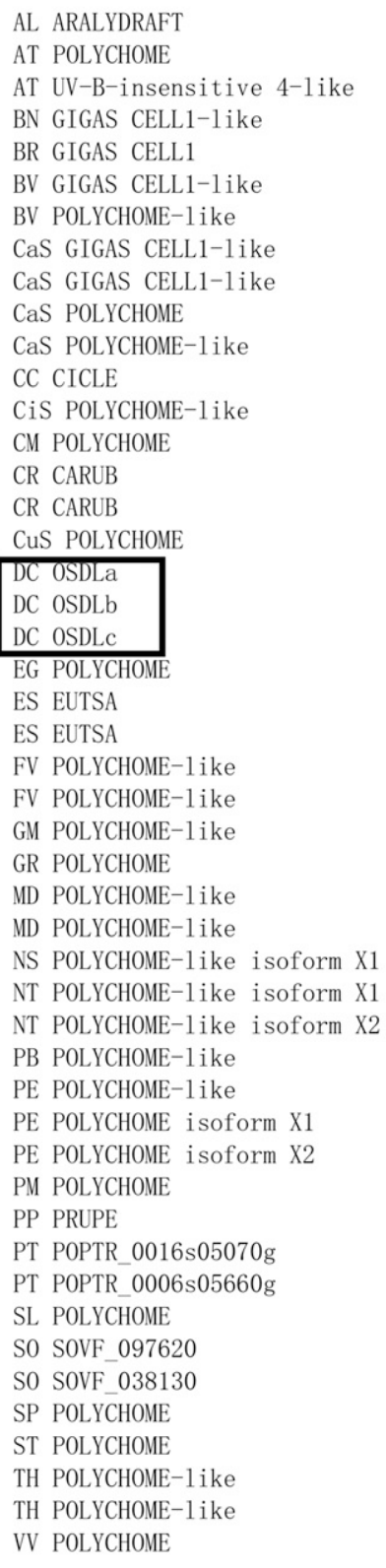

AL ARALYDRAFT

POLYCHOME

BN GIGAS CELL1-like

BR GIGAS CELL1

BV GIGAS CELL1-lik

BV POLYCHOME-like

CaS GIGAS CELL1-1ike

CaS POLYCHOME

CaS POLYCHOME-1ike

CiS POLYCHOME-1ike

CM POLYCHOME

CR CARUB

CuS POLYCHOME

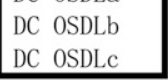

ES EUTSA

ES EUTSA

FV POLYCHONE-1ike

FV POLYCHOME-1ike

GI POLYCHOME-like

NT POLYCHOME-1ike isoform X1

NT POLYCHONE-1ike isoform X2

PE POLYCHOME isoform X1

PE POLYCHOME isoform X2

PM POLYCHOME

PP PRUPE

PT POPTR 0016s05070g

PT POPTR 0006s $05660 \mathrm{~g}$

SL POLYCHOME

TH POLYCHOME-like

VV POLYCHOME

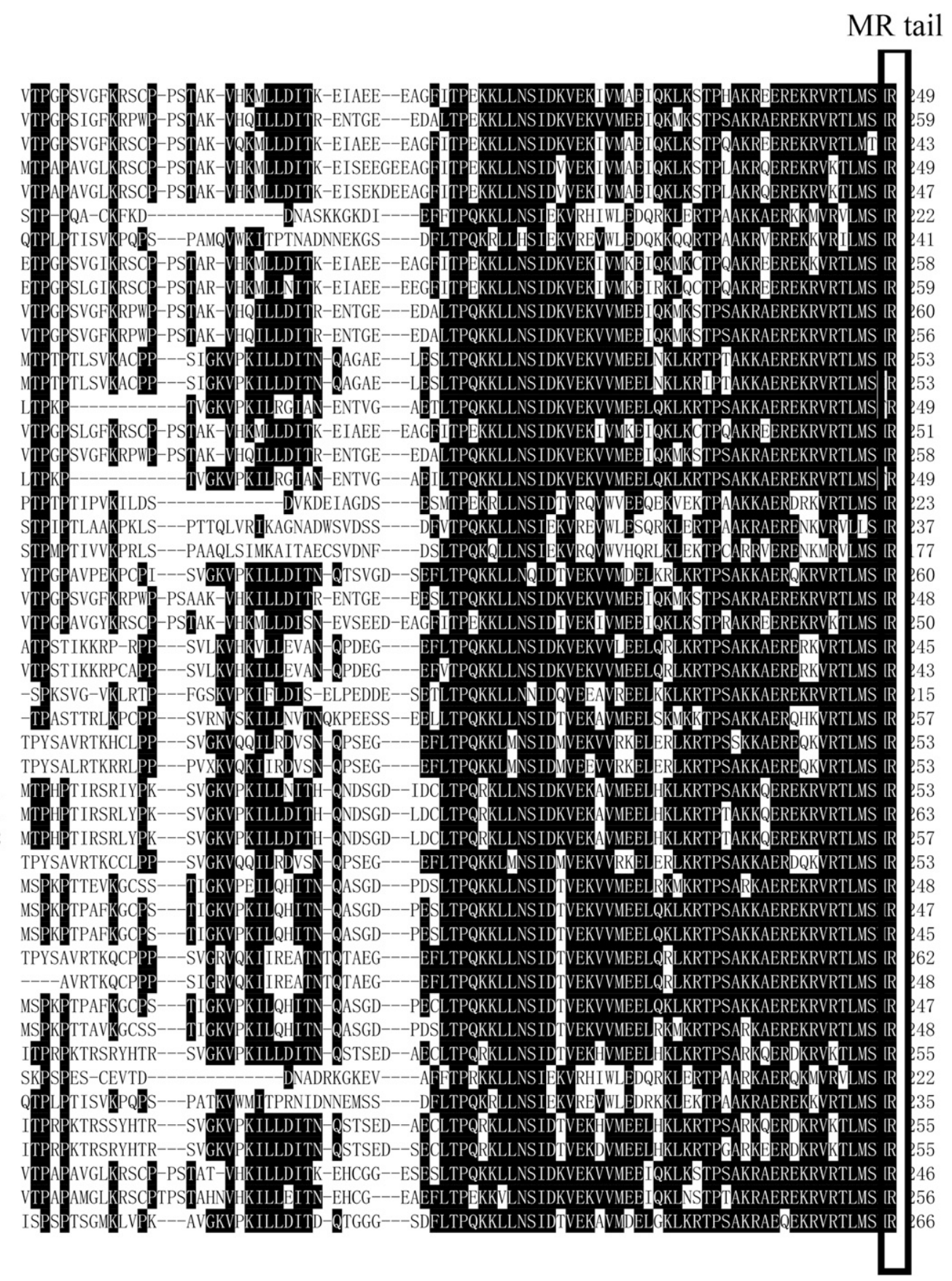

Fig. 2. (Continued) 
spores, called a tetrad (Fig. 1A). However, a low frequency of dyads (Fig. 1B) was observed, confirming that the diploid microspores are produced by a defect in meiotic chromosome segregation. Following chromosome condensation and crossover formation in pachytene and diakinesis (Fig. 1C and D), the metaphase I spindle aligns the bivalents at the equatorial plane (Fig. 1E) and subsequently, segregates the homologs toward the poles at anaphase I (Fig. 1F). Chromosomes decondensed at telophase $\mathrm{I}$ at the end of M I (Fig. 1G) and formed a dyad (Fig. 1H), strongly suggesting that dyad production is due to an absence of the second meiotic division.

Cloning and Characterization OF OSDL GENES IN CARNATION. $O S D 1$ is involved in the entry into both M I and M II in A. thaliana. osd1 mutants produce high levels of functional $2 \mathrm{n}$ gametes in the absence of a second meiotic division (d'Erfurth et al., 2009). Three different $O S D L$ cDNAs were isolated from prebolting buds of $D$. caryophyllus 'Nogalte'. After the composite cDNAs were obtained, the nucleotide sequences for the open reading frames (ORFs) of all these cDNAs were confirmed by cloning the complete (full-length) cDNAs by using PCR and sequencing. The three cDNAs were designated $O S D L a, O S D L b$, and $O S D L c$, and deposited in the NCBI database under accession numbers KX622764, KX622765, and KX622766, respectively.

ORFs were analyzed using the NCBI ORF Finder (Benson et al., 2004). The structures of the three $O S D L$ cDNAs are summarized in Supplemental Figs. 1-3, which include 5 '-flanking sequences, ORFs, and 3 '-flanking sequences. The cDNAs were $1180 \mathrm{bp}$ long for OSDLa, 1288 bp long for $O S D L b$, and 971 bp long for $O S D L c$. The predicted proteins consisted of 223 amino acids plus a 171-bp untranslated $5^{\prime}$ region and a 337-bp untranslated 3' region for OSDLa, 237 amino acids plus a 130 -bp untranslated $5^{\prime}$ region and a 444-bp untranslated $3^{\prime}$ region for $O S D L b$, and 177 amino acids plus a 2-bp untranslated 5 ' region and a $435-\mathrm{bp}$

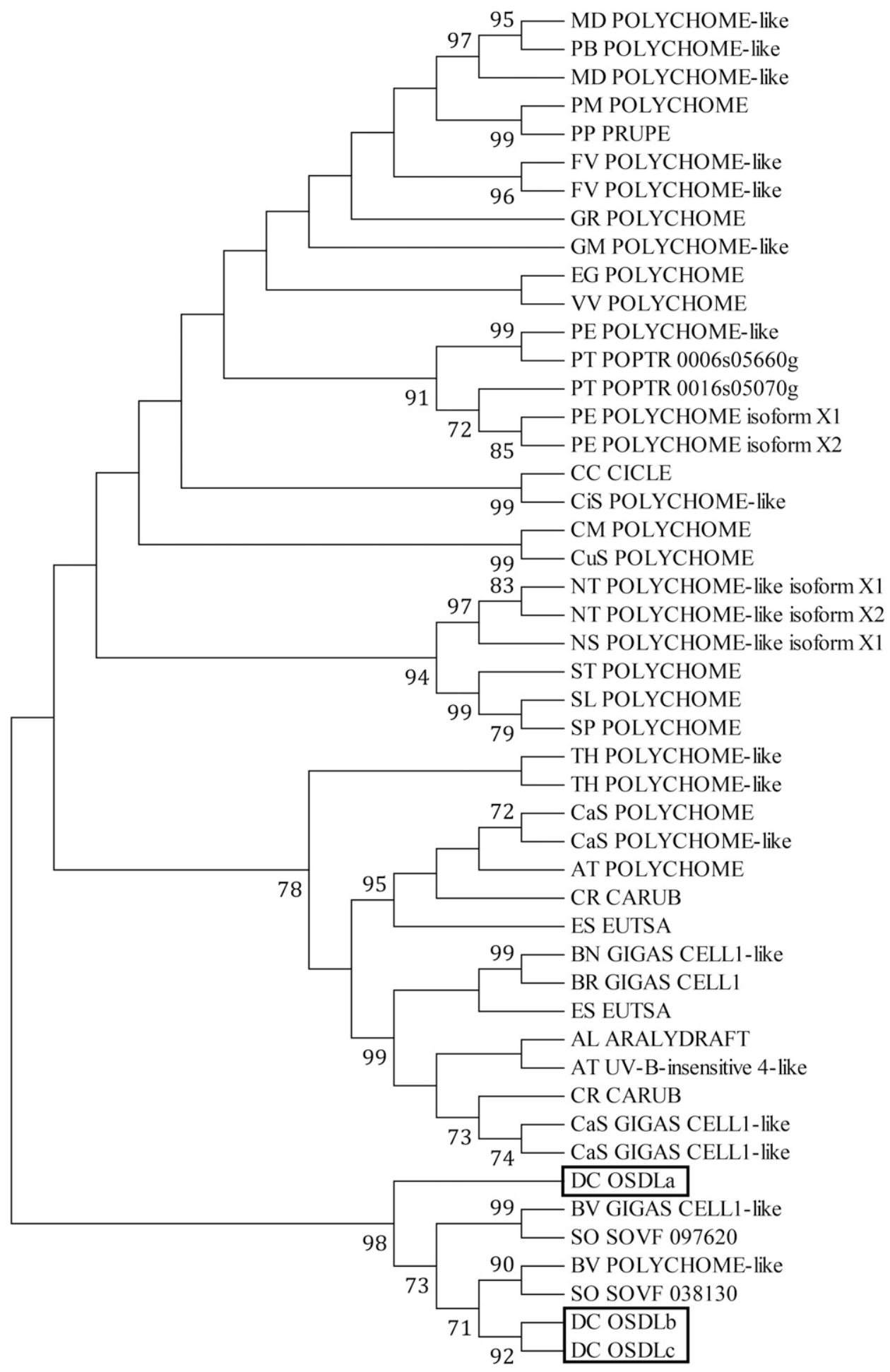

Fig. 3. Phylogenetic tree of plant omission of second division-like (OSDL) proteins. Maximum likelihood phylogenetic tree of $O S D L$ predicted proteins from 30 plant species: $\mathrm{AL}=$ Arabidopsis lyrata ssp. lyrata, $\mathrm{AT}=$ Arabidopsis thaliana, $\mathrm{BN}=$ Brassica napus, $\mathrm{BR}=$ Brassica rapa, $\mathrm{BV}=$ Beta vulgaris ssp. vulgaris, $\mathrm{CC}=$ Citrus clementina, $\mathrm{CM}=$ Cucumis melo, $\mathrm{CR}=$ Capsella rubella, $\mathrm{CaS}=$ Camelina sativa, $\mathrm{CiS}=$ Citrus sinensis, $\mathrm{DC}=$ Dianthus caryophyllus, $\mathrm{EG}=$ Eucalyptus grandis, $\mathrm{ES}=$ Eutrema salsugineum, $\mathrm{FV}=$ Fragaria vesca, $\mathrm{GM}=$ Glycine max, $\mathrm{GR}=$ Gossypium raimondii, $\mathrm{MD}=$ Malus domestica, $\mathrm{NS}=$ Nicotiana sylvestris, $\mathrm{NT}=$ Nicotiana tomentosiformis, $\mathrm{PB}=$ Pyrus bretschneideri, $\mathrm{PE}=$ Populus euphratica, $\mathrm{PM}=$ Prunus mume, $\mathrm{PP}=$ Prunus persica, $\mathrm{PT}=$ Populus trichocarpa, $\mathrm{SL}=$ Solanum lycopersicum, $\mathrm{SO}=$ Spinacia oleracea, $\mathrm{SP}=$ Solanum pennellii, $\mathrm{ST}=$ Solanum tuberosum, $\mathrm{TH}=$ Tarenay a hassleriana, $\mathrm{VV}=$ Vitis vinifera. 
untranslated $3^{\prime}$ region for $O S D L c$, corresponding to calculated molecular masses of 25.34, 25.95, and $19.95 \mathrm{kDa}$, respectively. Carnation OSDLs have two putative cellcycle-related domains which are putative $A P C / C$ degradation motifs: a D-box (RxxLxx, residues 104-109 for OSDLa, residues 111-116 for $O S D L b$, and residues 4853 for $O S D L c$ ) and a C-terminal MR-tail (Fig. 2). However, carnation OSDLs do not have a GxEN/KEN-box motif which is otherwise very well conserved across OSD1 homologues in plants (Cromer et al., 2012; Heyman et al., 2011).

The amino sequences of OSDLa have $47 \%$ and $48 \%$ similarity to $O S D L b$ and $O S D L c$, respectively, and $O S D L b$ has $55 \%$ similarity to $O S D L C$. A phylogenetic analysis was conducted by comparing the carnation OSDLS with 30 homeologous protein sequences from different plant species (Fig. 3). The three homologues of carnation OSDLs clustered on the same basal branch. A strong similarity was found between the amino acid sequences of $O S D L b$ and $O S D L c$, which clustered together as sisters.

COMPARISON OF TRANSCRIPT LEVELS OF OSDLS AMONG DIFFERENT TISSUES AND DIFFERENT DEVELOPMENTAL STAGES OF FLOWER BUDS. To test whether OSDLs were only expressed in the buds or also in other organs, we analyzed expression patterns. qRT-PCR analyses were performed to reveal expression levels in roots, leaves, stem, ovary, and buds. Expression analysis revealed that $O S D L s$ are expressed in many organs, including roots, leaves, stem, ovary, and buds (Fig. 4). Interestingly, OSDLa shows the highest expression in stage 2 buds in three carnation cultivars (Fig. 4A-C). The expression level of $O S D L a$ is significantly higher in stage 2 of buds in 'Promesa' (Fig. 4A) and in 'YunhongErhao' (Fig. 4C) than other stages of buds and tissues, and significantly higher in stage 1-3 of buds in 'Guernse Yellow' (Fig. 4B) than other stages of buds and tissues. OSDLb has a high level of expression in stage 1 buds and ovaries of 'Promesa' (Fig. 4D) and 'YunhongErhao' (Fig. 4F), and a high level of expression in stage 2 buds and ovaries of 'Guernse Yellow' (Fig. 4E). We also found that the expression level of $O S D L c$ in ovaries is higher than in other tissues (Fig. 4G-I).

\section{Discussion}

We cloned and characterized $O S D L$ genes in a diploid carnation and found three homoeologous genes that we called OSDLa, $O S D L b$, and $O S D L c$, which differ from those of A. thaliana. OSDLa and $O S D L b$ genes
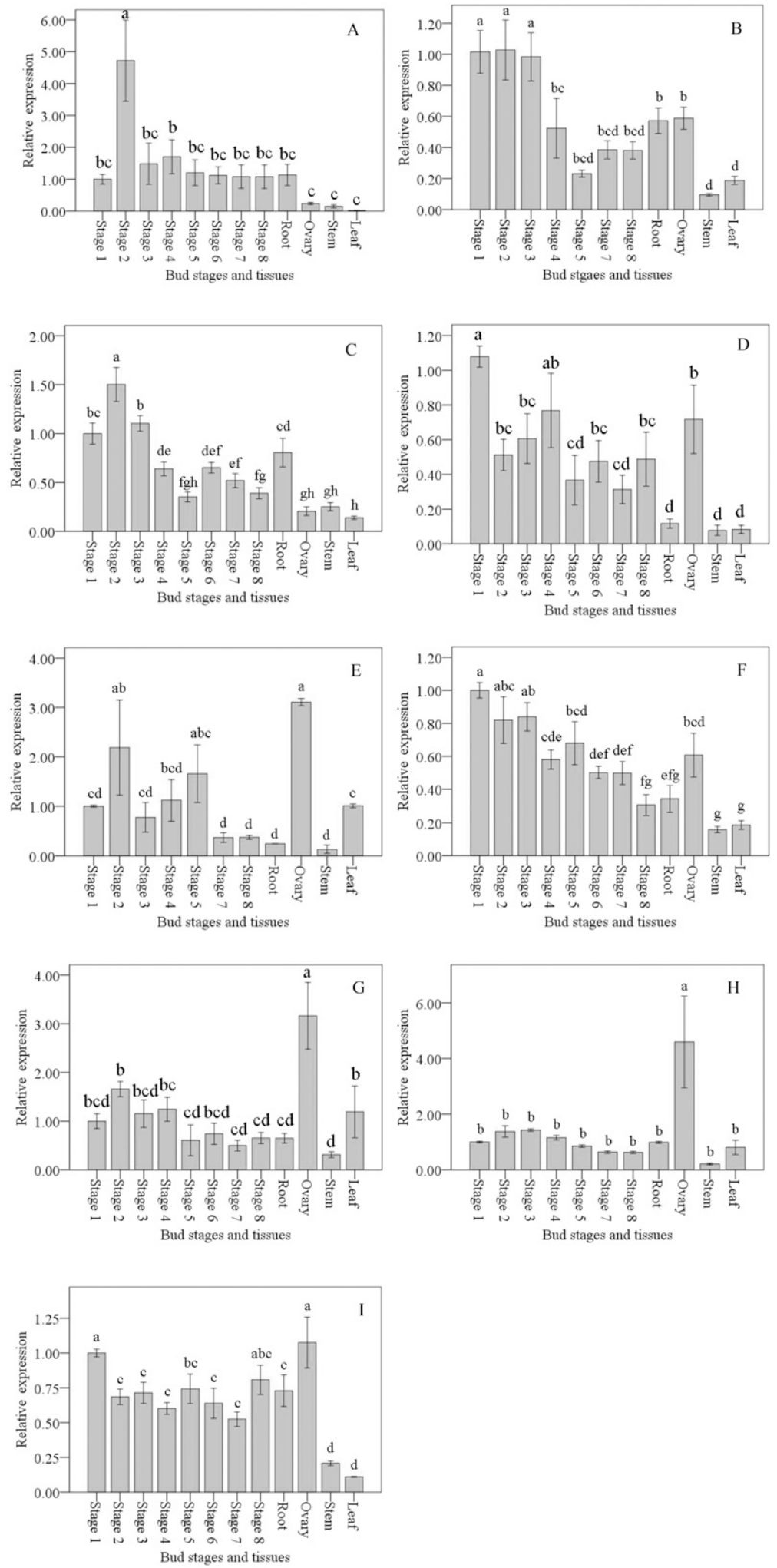

Fig. 4. Expression analysis of the OSD1-like gene in different bud stages and tissues of carnation. OSDLa gene expression analysis in carnation cultivars (A) Promesa, (B) Guernse Yellow, and (C) YunhongErhao; OSDLb gene expression analysis in carnation cultivars (D) Promesa, (E) Guernse Yellow, and (F) YunhongErhao; OSDLc gene expression analysis in carnation cultivars $(\mathbf{G})$ Promesa, (H) Guernse Yellow, and (I) YunhongErhao. Values are means \pm SE of three replicates. Different letters indicate significant differences $(P<0.05)$. Data were subjected to analysis of variance with the least significant difference method by statistical software package SPSS 18 (IBM Corp.). 
have longer sequences than does the $O S D L c$ gene. The evident feature of these $O S D L$ s proteins is the presence of D-box and MR-tail domains; however, the GxEN/KEN-box domain is lacking (Heyman et al., 2011). Thus, our results suggest that the GxEN/KEN-box might not be necessary for carnation $O S D L$ gene function.

Loss of OSD1 function was originally shown to cause defects in the second mitotic division during the meiotic cell cycle, thus leading to the production of diploid male and female gametes in $A$. thaliana (d'Erfurth et al., 2009), suggesting that OSD1 gene was expressed in buds. Bao and Hua (2014) found that megaspores cannot complete mitosis to develop into a functional female gametophyte in osd 1 uvi4 genotypes, and indicating the OSD1 and UVI4 together are essential for zygote development as well. UVI4 is expressed during the mitotic cell cycle, peaking at the G1-to-S transition, whereas the expression of OSD1 peaks at the G2-to-M transition (Heyman et al., 2011). UVI4 plays an important role in determining the meristem size in roots and the cell number and size in leaves (Heyman et al., 2011). The level of OSD1 is critical in controlling ploidy levels in leaf cells, most likely through regulating endoreduplication (Bao and Hua, 2014). From the expression patterns we observed in carnation, $O S D L$ genes maintain continuous expression within buds and many other tissues, suggesting a role in somatic growth. OSDLa was most highly expressed in stage 2 buds of 'Promesa', 'Guernse Yellow', and 'YunhongErhao'. This suggests that OSDLa may be involved in male meiosis. OSDLb has a high level of expression in both stage 1 and stage 2 buds and in ovary tissues in carnation cultivars. OSDLb may be associated with both pollen and ovary development. The expression level of $O S D L c$ in ovaries is highest compared with other tissues, suggesting $O S D L c$ probably functions in female gametogenesis, meiotic progression, or both. However, these hypotheses regarding the function of $O S D L s$ in carnation have not been confirmed. In future studies, we plan to focus on the function and the regulation network of OSDLs.

\section{Conclusion}

Diploid gamete formation occurred in carnation. Analysis of chromosome behavior at meiosis indicated that $2 \mathrm{n}$ male gamete formation is probably attributable to the absence of the second meiotic division. We cloned $O S D L$ genes in carnation for the first time by using RACE technology and have identified three homoeologous genes, which can be distinguished from OSD1 in A. thaliana. The evident feature of these $O S D L$ s proteins is the absence of the GxEN/KEN-box domain, suggesting that the GxEN/KEN-box is probably not necessary for gene function in carnation. Our analyses of expression levels of $O S D L$ indicated that $O S D L s$ in carnation may play roles in male meiosis and ovary development. These findings have potential applications in fundamental polyploidization research and carnation breeding programs.

\section{Literature Cited}

Bao, Z. and J. Hua. 2014. Interaction of CPR5 with cell cycle regulators UVI4 and OSD1 in Arabidopsis. PLoS One 9:e100347.

Benson, D.A., K.M. Ilene, D.J. Lipman, O. James, and D.L. Wheeler. 1997. GenBank. Nucleic Acids Res. 25:1-6.

Benson, D.A., I. Karschmizrachi, D.J. Lipman, J. Ostell, and D.L. Wheeler. 2004. GenBank: Update. Nucl. Acids Res. 32:D23-D26.
Bretagnolle, F. and J. Thompson. 1995. Gametes with the somatic chromosome number: Mechanisms of their formation and role in the evolution of autopolyploid plants. New Phytol. 129:1-22.

Brownfield, L. and C. Köhler. 2011. Unreduced gamete formation in plants: Mechanisms and prospects. J. Expt. Bot. 62:1659-1668.

Cromer, L., J. Heyman, S. Touati, H. Harashima, E. Araou, C. Girard, C. Horlow, K. Wassmann, A. Schnittger, and L. De Veylder. 2012. OSD1 promotes meiotic progression via $\mathrm{APC} / \mathrm{C}$ inhibition and forms a regulatory network with TDM and CYCA1;2/TAM. PLoS Genet. 8:e1002865.

d'Erfurth, I., L. Cromer, S. Jolivet, C. Girard, C. Horlow, Y. Sun, J.P.C. To, L.E. Berchowitz, G.P. Copenhaver, and R. Mercier. 2010. The CYCLIN-A CYCA1;2/TAM is required for the meiosis I to meiosis II transition and cooperates with OSD1 for the prophase to first meiotic division transition. PLoS Genet. 6:e1000989.

d'Erfurth, I., S. Jolivet, N. Froger, O. Catrice, M. Novatchkova, and R. Mercier. 2009. Turning meiosis into mitosis. PLoS Biol. 7:e1000124. de Gramont, A. and O. Cohen-Fix. 2005. The many phases of anaphase. Trends Biochem. Sci. 30:559-568.

Francis, D. 2007. The plant cell cycle -15 years on. New Phytol. 174:261-278.

Furuno, N., M. Nishizawa, K. Okazaki, H. Tanaka, J. Iwashita, N. Nakajo, Y. Ogawa, and N. Sagata. 1994. Suppression of DNA replication via Mos function during meiotic divisions in Xenopus oocytes. EMBO J. 13:2399-2410.

Futcher, B. 2008. Cyclins in meiosis: Lost in translation. Dev. Cell 14:644-645.

Gasteiger, E., A. Gattiker, C. Hoogland, I. Ivanyi, R.D. Appel, and A. Bairoch. 2003. ExPASy: The proteomics server for in-depth protein knowledge and analysis. Nucl. Acids Res. 31:3784-3788.

Gómez-Rodríguez, V.M., B. Rodríguez-Garay, and R. Barba-Gonzalez. 2012. Meiotic restitution mechanisms involved in the formation of 2n pollen in Agave tequilana Weber and Agave angustifolia Haw. Springerplus 1(17):1-7.

Heyman, J., H. Van den Daele, K. De Wit, V. Boudolf, B. Berckmans, A. Verkest, C.L.A. Kamei, G. De Jaeger, C. Koncz, and L. De Veylder. 2011. Arabidopsis ultraviolet-b-insensitive4 maintains cell division activity by temporal inhibition of the anaphase-promoting complex/cyclosome. Plant Cell 23:4394-4410.

Inzé, D. and L. De Veylder. 2006. Cell cycle regulation in plant development. Annu. Rev. Genet. 40:77-105.

Iwabuchi, M., K. Ohsumi, T.M. Yamamoto, W. Sawada, and T. Kishimoto. 2000. Residual Cdc2 activity remaining at meiosis I exit is essential for meiotic $\mathrm{M}-\mathrm{M}$ transition in Xenopus oocyte extracts. EMBO J. 19:4513-4523.

Iwata, E., S. Ikeda, S. Matsunaga, M. Kurata, Y. Yoshioka, M.C. Criqui, P. Genschik, and M. Ito. 2011. GIGAS CELL1, a novel negative regulator of the anaphase-promoting complex/cyclosome, is required for proper mitotic progression and cell fate determination in Arabidopsis. Plant Cell 23:4382-4393.

Marston, A.L. and A. Amon. 2004. Meiosis: Cell-cycle controls shuffle and deal. Nat. Rev. Mol. Cell Biol. 5:983-997.

Menges, M., S.M. De Jager, W. Gruissem, and J.A. Murray. 2005. Global analysis of the core cell cycle regulators of Arabidopsis identifies novel genes, reveals multiple and highly specific profiles of expression and provides a coherent model for plant cell cycle control. Plant J. 41:546-566.

Mok, D. and S. Peloquin. 1975. Three mechanisms of $2 \mathrm{n}$ pollen formation in diploid potatoes. Can. J. Genet. Cytol. 17:217-225.

Otto, S.P. 2007. The evolutionary consequences of polyploidy. Cell 131:452-462.

Otto, S.P. and J. Whitton. 2000. Polyploid incidence and evolution. Annu. Rev. Genet. 34:401-437.

Pesin, J.A. and T.L. Orr-Weaver. 2008. Regulation of APC/C activators in mitosis and meiosis. Annu. Rev. Cell Dev. Biol. 24:475-499. 
Ramanna, M. and E. Jacobsen. 2003. Relevance of sexual polyploidization for crop improvement - A review. Euphytica 133:3-8.

Ramanna, M.S. 1983. First division restitution gametes through fertile desynaptic mutants of potato. Euphytica 32:337-350.

Stern, B.M. 2003. FEARless in meiosis. Mol. Cell 11:1123-1125.

Tamura, K., D. Peterson, N. Peterson, G. Stecher, M. Nei, and S. Kumar. 2011. MEGA5: Molecular evolutionary genetics analysis using maximum likelihood, evolutionary distance, and maximum parsimony methods. Mol. Biol. Evol. 28:2731-2739.

Tanase, K., C. Nishitani, H. Hirakawa, S. Isobe, S. Tabata, A. Ohmiya, and T. Onozaki. 2012. Transcriptome analysis of carnation (Dianthus caryophyllus L.) based on next-generation sequencing technology. BMC Genomics 13:1-11.
Teoh, S. 1984. Polyploid spore formation in diploid orchid species. Genetica 63:53-59.

Veilleux, R. 1985. Diploid and polyploid gametes in crop plants: Mechanisms of formation and utilization in plant breeding. Plant Breed. Rev. 3:253-288.

Zhou, X., M. Gui, J. Wang, M. Chen, J. Long, Z. Lu, J. Song, and X. Mo. 2012. Pollen tube growth after crossing between different ploids Dianthus caryophyllus by fluorescence microscopy and seed setting. Acta Bot. Boreali-Occidential Sin. 32:67-74.

Zhou, X., X. Mo, M. Gui, X. Wu, Y. Jiang, L. Ma, Z. Shi, Y. Luo, and W. Tang. 2015. Cytological, molecular mechanisms and temperature stress regulating production of diploid male gametes in Dianthus caryophyllus L. Plant Physiol. Biochem. 97:255263. 


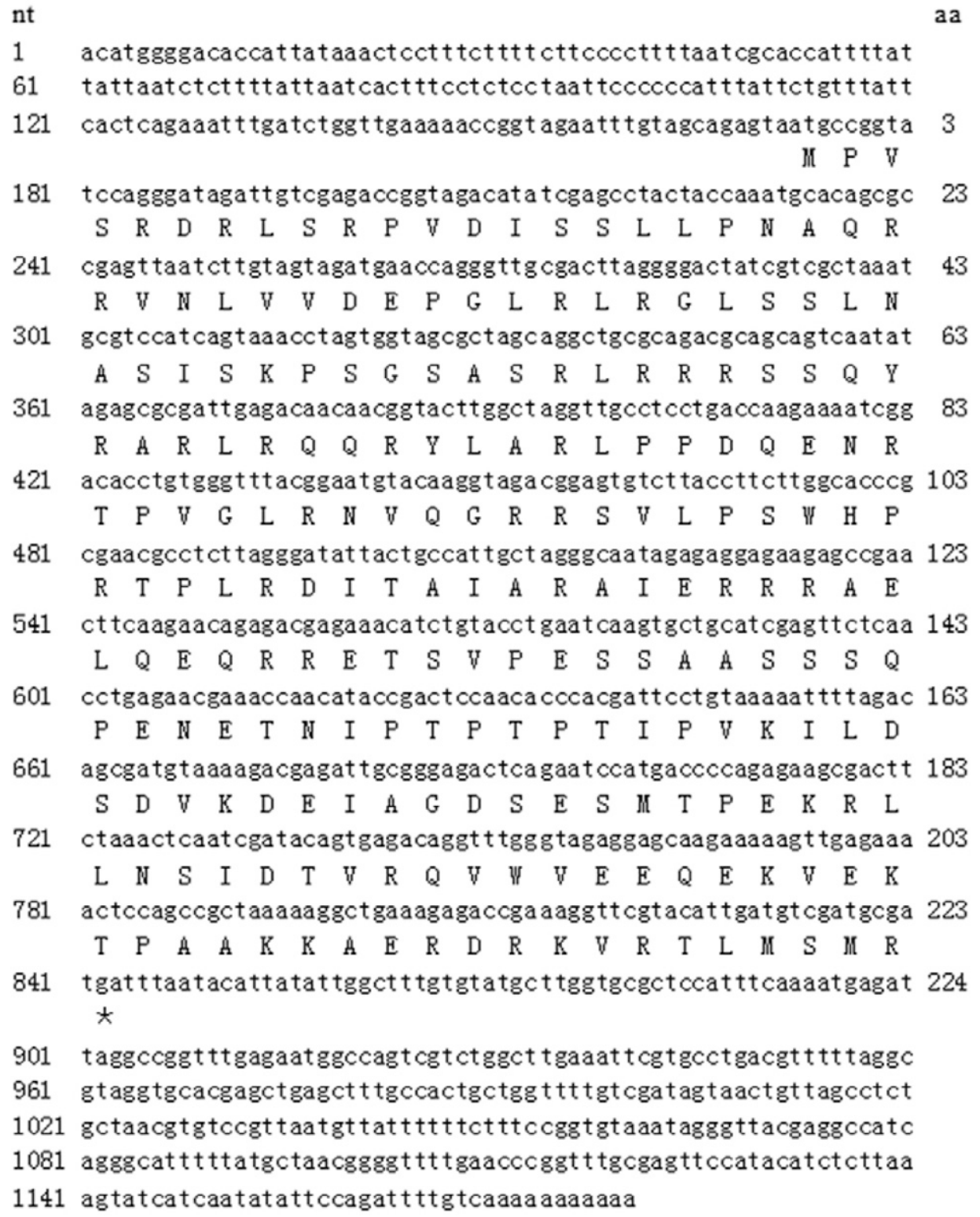

Supplemental Fig. 1. Open reading frame and presumed amino acid sequences of OSDLa in carnation. 


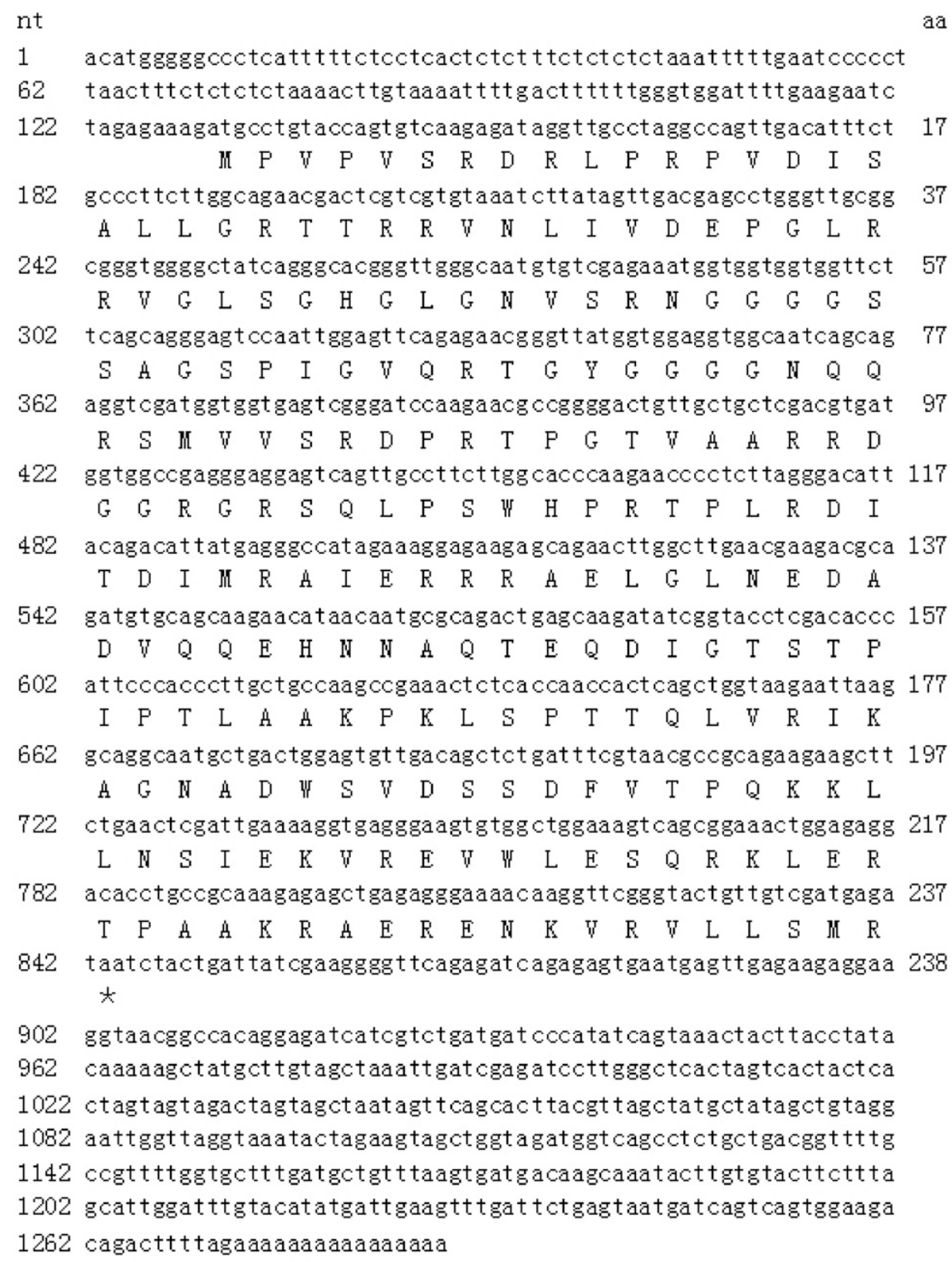

Supplemental Fig. 2. Open reading frame and presumed amino acid sequences of $O S D L b$ in carnation. 


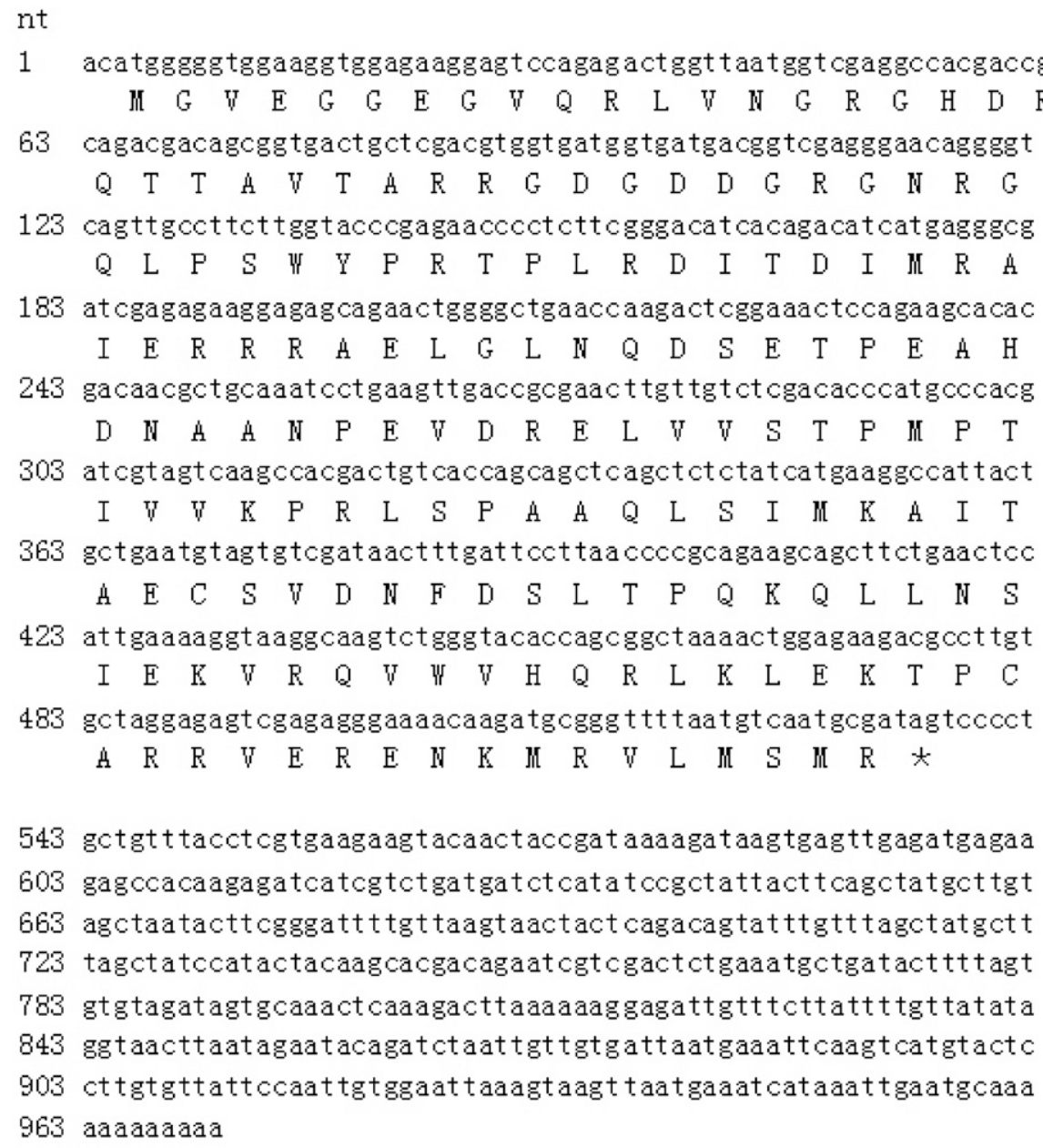

Supplemental Fig. 3. Open reading frame and presumed amino acid sequences of $O S D L c$ in carnation. 
Supplemental Table 1. Primers used in this study [forward primer (FP), reverse primer (RP)].

\begin{tabular}{ll}
\hline Name & \multicolumn{1}{c}{${\text { Primer sequence } 5^{\prime} \text { to } 3^{\prime}}^{\prime}$} \\
OSDL1a-5Ra-364R & RP CCTAGCCAAGTACCGTTGTTGTCTCA \\
OSDL1a-82-length-F & FP ACTTTCCTCTCCTAATTCC \\
OSDL1b-103-length-F & FP CCATTTATTCTGTTTATTCACTCA \\
OSDL1b-138-length-F & RP GCTTCTTCTGCGGCGTTACGA \\
OSDL1b-147-length-F & FP ATGCCTGTACCAGTGTCAAG \\
OSDL1c-5R-468R & FP ATGCCTGTACCAGTGTCAAGAGATAGGT \\
OSDL1c-328R & RP CTGTCGTGCTTGTAGTATGG \\
OSDL1c-69-length-F & RP GTGGCTCTTCTCATCTCAA \\
OSDL1c-113-length-F & FP ACAGCGGTGACTGCTCGACGTG \\
GAPDH 579-F & FP GAACAGGGGTCAGTTGCCTTCTTGGTA \\
GAPDH 788-R & FP CAGTGGGCACACGGAAAGC \\
OSDL1a-422F & RP TGGCATCGTTGAGGGTCT \\
OSDL1a-656R & FP CGGAGTGTCTTACCTTCTT \\
OSDL1b-99-F & RP CTCTCGCAATCTCGTCTT \\
OSDL1b-223-R & FP CTCTCACCAACCACTCAG \\
OSDL1c-133F & RP CACACTTCCCTCACCTTT \\
OSDL1c-270-R & FP CTGAACTCCATTGAAAAGGTAAGGC \\
\hline
\end{tabular}

